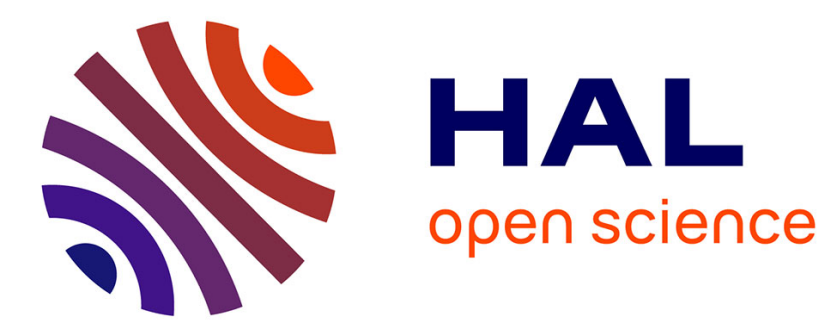

\title{
Couches de haute résistivité dans InP obtenues par implantation ionique
}

\author{
P.N. Favennec, H. L'Haridon, M. Salvi, M. Gauneau
}

\section{To cite this version:}

P.N. Favennec, H. L'Haridon, M. Salvi, M. Gauneau. Couches de haute résistivité dans InP obtenues par implantation ionique. Revue de Physique Appliquée, 1984, 19 (3), pp.191-195. 10.1051/rphysap:01984001903019100 . jpa-00245182

\section{HAL Id: jpa-00245182 https://hal.science/jpa-00245182}

Submitted on 1 Jan 1984

HAL is a multi-disciplinary open access archive for the deposit and dissemination of scientific research documents, whether they are published or not. The documents may come from teaching and research institutions in France or abroad, or from public or private research centers.
L'archive ouverte pluridisciplinaire HAL, est destinée au dépôt et à la diffusion de documents scientifiques de niveau recherche, publiés ou non, émanant des établissements d'enseignement et de recherche français ou étrangers, des laboratoires publics ou privés. 
Classification

Physics Abstracts

$61.70 \mathrm{~T}-61.80 \mathrm{~J}-72.90 \mathrm{E}$

\title{
Couches de haute résistivité dans InP obtenues par implantation ionique
}

\author{
P. N. Favennec, H. L'Haridon, M. Salvi et M. Gauneau \\ Division ICM/TOH, CNET-Lannion B, 22301 Lannion Cedex, France
}

(Reçu le 25 juillet 1983, révisé le 14 octobre, accepté le 18 novembre 1983)

\begin{abstract}
Résumé. - Des ions de différentes natures $\left(\mathrm{H}^{+}, \mathrm{B}^{+}, \mathrm{O}^{+}, \mathrm{Ne}^{+}, \mathrm{A}^{+}, \mathrm{Fe}^{+}\right)$ont été implantés dans l'InP de type $\mathrm{n}$ pour étudier les conditions d'obtention de couches de haute résistivité. Quel que soit l'ion incident, et pour de l'InP recuit, la résistivité varie de la même manière en fonction de la concentration d'ions implantés. L'efficacité de compensation des ions est montrée être dépendante de leur masse. Une température de recuit de $650^{\circ} \mathrm{C}$ est nécessaire pour recuire les défauts d'implantation responsables de la compensation. Les ions d'oxygène et bore semblent inactifs dans le matériau InP après recuit à haute température. Par contre, l'implantation de fer entraîne la formation de couches de haute résistivité $\left(e \gtrsim 10^{7} \Omega \mathrm{cm}\right)$ après recuit à haute température, mais cette haute résistivité ne serait pas uniquement due à l'impureté fer.
\end{abstract}

\begin{abstract}
Various ions $\left(\mathrm{H}^{+}, \mathrm{B}^{+}, \mathrm{O}^{+}, \mathrm{Ne}^{+}, \mathrm{A}^{+}, \mathrm{Fe}^{+}\right)$have been implanted in n-type InP for studying the conditions to obtain high resistivity layers. For unannealed samples, the shape of the resistivity variation versus the ion dose is independent of the ion nature. The compensation efficiency of the ions is shown to be dependent of the ion mass. The defects responsible of the high resistivity are totally annealed at $650{ }^{\circ} \mathrm{C}$. Oxygen and boron ions seem inactive in InP after annealing of high temperature. Iron implantations entrain a formation of high resistivity layers $\left(e \gtrsim 10^{7} \Omega \mathrm{cm}\right.$ ) after an anneal at high temperature, but this high resistivity should not be due, solely, to the iron impurity.
\end{abstract}

\section{Introduction.}

Dans l'arséniure de gallium et $\mathrm{AlGaAs}$, de nombreux travaux ont montré que des couches de haute résistivité peuvent être obtenues par implantation ionique soit pour du matériau non recuit par utilisation des défauts de bombardement [1 à 3] soit pour du matériau recuit à haute température par utilisation des propriétés de certaines impuretés telles que le bore [4 à 6], l'oxygène $[7,8]$ et le chrome [9]. Dans le phosphure d'indium, quelques résultats publiés montrent que les défauts d'implantation sont aussi des centres de compensation pouvant entraîner une forte augmentation de la résistivité des couches implantées [10 à 13]. Nous allons étudier les possibilités de formation, par implantation ionique, de couches d'InP à forte résistivité et montrer plus particulièrement quel est le comportement du bore, de l'oxygène et du fer dans le matériau InP de type $n$.

\section{Compensation par les défauts d'implantation.}

La détermination de la résistivité des couches implantées est faite à partir d'échantillons épitaxiés n/SI [14]. Ceux-ci sont prédécoupés sous la forme de barreaux, leurs extrémités ne sont pas implantées, elles sont recouvertes de métallisations Au-Ge épaisses dont le rôle est de faire un contact ohmique sur la couche $n$ et de faire masque aux faisceaux d'implantation. Seules les parties centrales, non masquées par les métallisations, sont implantées. Les conditions d'implantation (énergies et doses) sont telles que la concentration $\left(\mathrm{en} \mathrm{cm}^{-3}\right)$ des ions implantés, $n_{\text {ion }}$, reste constante de la surface au substrat semi-isolant (typiquement l'épaisseur de la couche épitaxiée faisait $1 \mu \mathrm{m})$. Avec de tels échantillons, connaissant les paramètres géométriques, une simple mesure de résistance entre les deux extrémités du barreau nous permet d'accéder à la résistivité moyenne de la couche implantée.

Quelle que soit la nature de l'ion incident, on constate après implantation une augmentation de résistivité de la couche InP bombardée n'ayant subi aucun traitement thermique. Nous présentons dans la figure 1 pour le cas du bore implanté, la variation de la résistivité de la couche bombardée en fonction de la concentration de bore introduite par implantation ionique. Pour cette expérience, les échantillons étaient des couches InP épitaxiées de type $n$ sur substrat semi-isolant. 


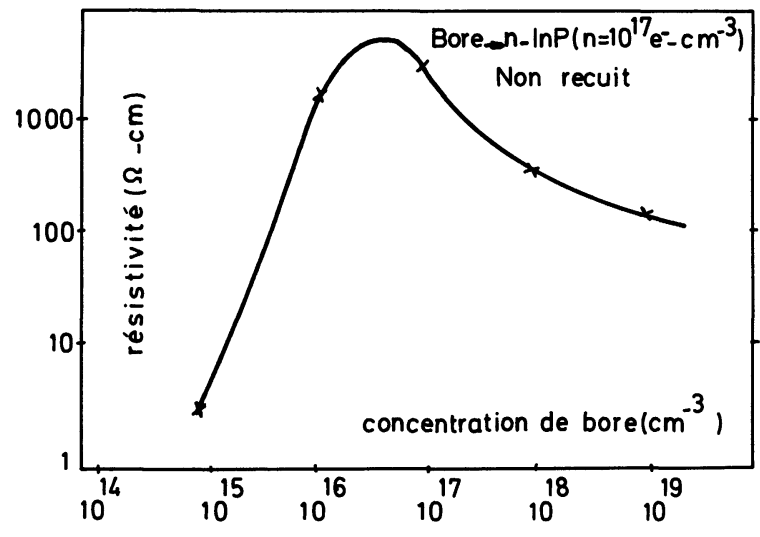

Fig. 1. - Variation de la résistivité avec la concentration de bore implanté.

[Resistivity variation versus implanted boron concentration.]

Les caractéristiques de la couche épitaxiale étaient : $n=10^{17} \mathrm{e}^{-} \cdot \mathrm{cm}^{-3} e=2 \times 10^{-2} \Omega$.cm, épaisseur $=$ $1 \mu \mathrm{m}$. Dans ces échantillons, nous avions procédé à des multi-implantations de bore dont les énergies et les doses étaient calculées pour avoir une concentration constante de la surface à l'interface épitaxiesubstrat semi-isolant. La résistivité de tels échantillons implantés croît aux faibles concentrations de bore, passe par un maximum $\left(e \operatorname{maxi}=5 \times 10^{3} \Omega \mathrm{cm}\right)$ pour une concentration de bore de $3,2 \times 10^{16}$ bore $\mathrm{cm}^{3}$ ), puis décroît ensuite quand les concentrations de bore introduites sont plus importantes.

Si on change la nature de l'impureté implantée, en gardant la condition d'avoir une concentration constante de la surface au substrat semi-isolant, la forme de la courbe résistivité en fonction de la concentration d'impuretés implantées reste identique, mais la concentration nécessaire pour avoir la résistivité maximale, qui est toujours égale à $5 \times 10^{3} \Omega \mathrm{cm}$, est modifiée en fonction de la masse d'ion incident. Plus l'ion incident est lourd, plus la concentration nécessaire est faible. Appelons efficacité de compensation $K$, le rapport entre la concentration en porteurs, $n$, de l'échantillon avant implantation et la concentration d'impuretés implantées, $n_{\mathrm{l}}$, nécessaires pour avoir la résistivité maximale $\left(K=n / n_{1}\right)$. Nous montrons dans la figure 2, la variation de cette efficacité de compensation, c'est-à-dire la diminution de porteurs par ion incident, en fonction de la masse de l'impureté implantée $(M=1,11,16,28,32)$. $K$ croît avec la masse, et comme dans le GaAs, cette efficacité de compensation est proportionnelle à l'énergie déposée par chocs nucléaires, ce qui traduit qu'elle est induite par les atomes déplacés de la matrice InP. A partir de la connaissance $K$, nous avons déduit une courbe générale (Fig. 3) de la variation de résistivité pour de l'InP de type $\mathbf{n}$ bombardé mais non recuit, en abscisse nous avons $K \frac{n_{\text {ion }}}{n}$, où $n_{\text {ion }}$ est la concentration d'ions implantés en $\mathrm{cm}^{-3}$. Sur la courbe, quelques exemples expéri- mentaux sont reportés pour l'hydrogène, le bore et l'oxygène et nous notons que la valeur de la résistivité ne dépend que du rapport $K \frac{n_{\text {ion }}}{n}$. Ces résultats, montrés dans les figures 2 et 3 , indiquent bien que la

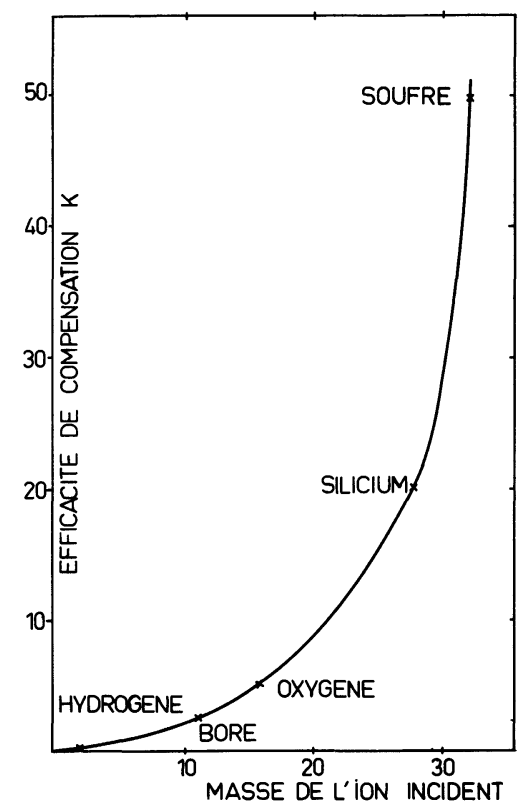

Fig. 2. - Efficacité de compensation des ions implantés en fonction de leur masse, pour de l'InP de type n implanté et non recuit.

[Compensation efficiency of the implanted ions versus ion mass, for implanted and unannealed n-type InP.]

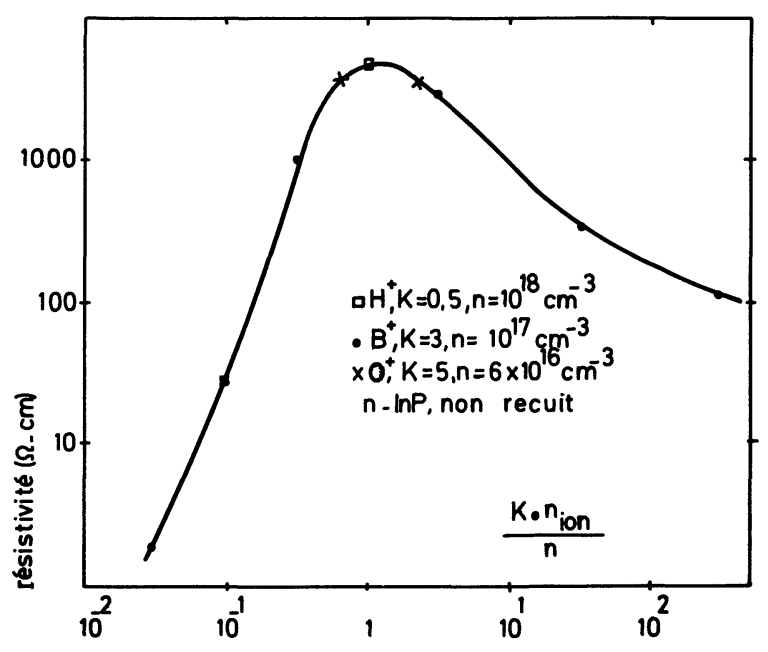

Fig. 3. - Variation de la résistivité du phosphure d'indium de type $n$ implanté en fonction de $K n_{\text {ion }} / n$ où $K$ est l'efficacité de compensation de l'ion implanté, $n_{\text {ion }}$ est la concentration en $\mathrm{cm}^{-3}$ des ions implantés et $n$ est la concentration en porteurs de l'InP avant implantation.

[Resistivity variation for implanted n-type InP versus $K n_{\text {ion }} / n, K$ is the compensation efficiency, $n_{\text {ion }}$ is the implanted ions concentration and $n$ is the electron carrier concentration of the substrate.] 
compensation obtenue après bombardement ne dépend uniquement que du nombre d'atomes (dépendance avec la masse de l'ion) de la matrice déplacés lors des chocs nucléaires et non de la nature de l'impureté implantée.

\section{Variations de la résistivité avec la température.}

Nous avons étudié la variation de la résistivité des couches implantées en fonction de la température de recuit. Pour les manipulations décrites ici, les échantillons d'InP étaient protégés pour tous les recuits (de $100{ }^{\circ} \mathrm{C}$ à $750^{\circ} \mathrm{C}$ ) par un film de $2000 \AA \mathrm{de}^{\mathrm{Si}} \mathrm{Si}_{3} \mathrm{~N}_{4}$ obtenu par pulvérisation réactive. Nous avons vérifié qu'un tel film de passivation protégeait effectivement bien, jusqu'à $750^{\circ} \mathrm{C}$, un échantillon de référence non implanté, au moins au sens de la résistivité du matériau (voir courbe de référence de la figure 4). Pour les températures supérieures à $750^{\circ} \mathrm{C}$, le film de $\mathrm{Si}_{3} \mathrm{~N}_{4}$ se craquèle et le matériau se dégrade (forte augmentation de sa résistivité), aussi il nous est impossible d'étudier l'effet de l'implantation pour de telles températures de recuit. Sur la figure 4, nous traçons, pour diverses concentrations de bore implantées, la variation de la résistivité avec la température de recuit. Le temps de chaque recuit est de $30 \mathrm{~min}$. A partir de ce réseau de courbes, nous distinguons deux catégories d'implantation.

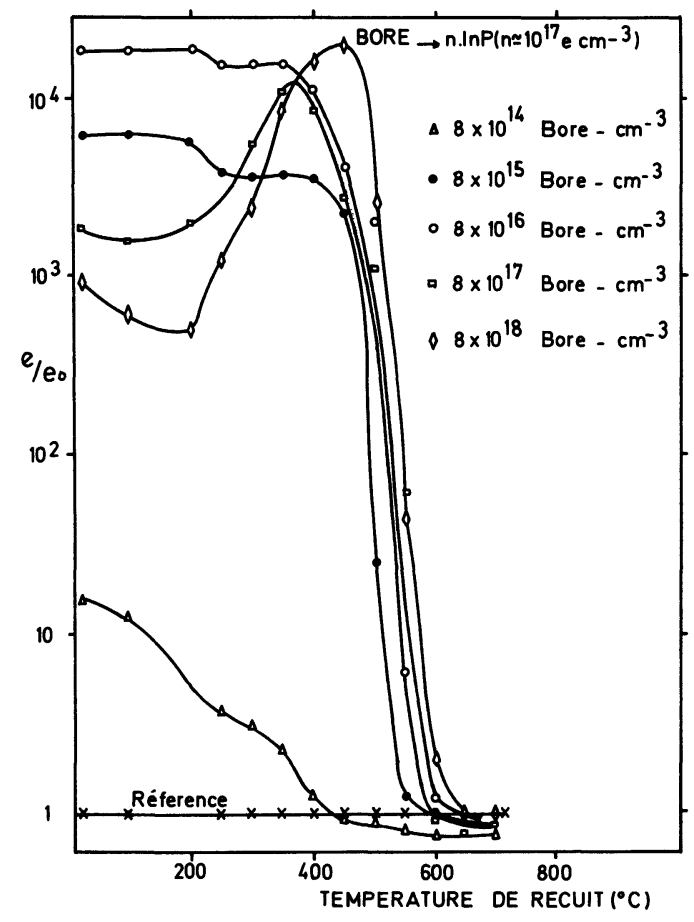

Fig. 4. - Variation de la résistivité d'InP implanté de type $n$ pour des recuits isochrones. Le temps de recuit est de $30 \mathrm{~min}$ est la résistivité initiale de l'InP.

[Resistivity variation versus the isochronal annealing temperature. $e_{0}$ is the initial resistivity and the duration of the annealing is $30 \mathrm{~min}$.]
1. Pour les faibles concentrations implantées, les cas où $K \frac{n_{\text {ion }}}{n} \leqslant 1$, la résistivité décroît avec la température. Deux stades de recuit sont visibles : $T \simeq 225^{\circ} \mathrm{C}$ et $T \simeq 500^{\circ} \mathrm{C}$.

2. Pour les fortes concentrations implantées, les cas où $K \frac{n_{\text {ion }}}{n}>1$, les courbes de recuits isochrones sont plus complexes. La résistivité décroît d'abord pour les températures de recuit faibles, puis elle croît ensuite jusqu'à des valeurs proches de la résistivité maximale ( $e$ maxi $=5 \times 10^{3} \Omega \mathrm{cm}$ ) pour des températures de l'ordre de $400^{\circ} \mathrm{C}$ à $500^{\circ} \mathrm{C}$, puis elle décroît très rapidement pour les températures supérieures.

Les températures de recuit nécessaires pour retrouver la résistivité initiale de l'échantillon dépendent de la concentration d'impuretés implantées (Fig. 5) et donc de la densité de défauts introduites par l'implantation. Dans le domaine des faibles concentrations d'impuretés implantées, la température croît avec la dose, puis pour les fortes concentrations, une température de $650^{\circ} \mathrm{C}$ est nécessaire pour retrouver la résistivité initiale dans la couche d'InP implantée. Des recuits supplémentaires à $700^{\circ} \mathrm{C}$ de ces échantillons guéris n'entraînent pas de modification de la résistivité de la couche d'InP bombardée et recuite. Des résultats analogues, à ceux montrés dans le cas du bore, ont été obtenus pour des implantations de néon, d'argon et d'oxygène. Pour ces quatre impuretés, il y a une guérison totale à $700{ }^{\circ} \mathrm{C}$. Alors, les centres de compensation induits par le déplacement des atomes de la matrice lors des chocs nucléaires ont totalement disparus, et les impuretés bore, oxygène, néon et argon semblent toutes, les quatre, être inactives dans le matériau InP. Ces résultats montrent que le bore et l'oxygène ont un comportement différent dans le matériau GaAs $[4,7]$ et dans le matériau InP, puisque danş le GaAs le matériau implanté böre ou oxygène et recuit à haute température est semi-isolant.

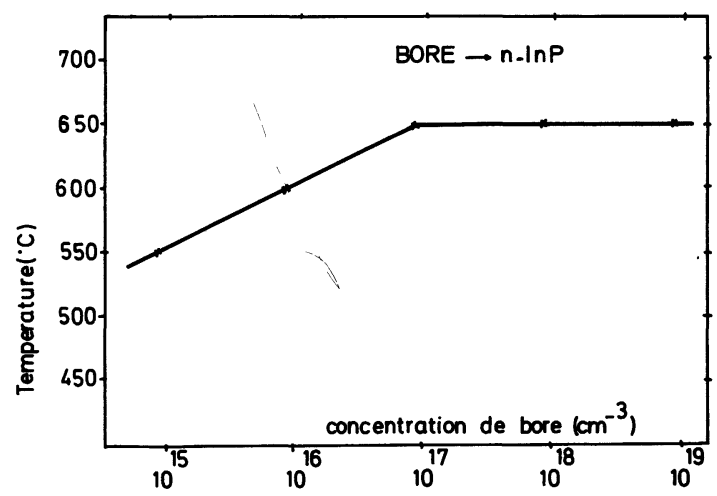

Fig. 5. - Température de recuit nécessaire pour avoir guérison totale des défauts d'implantation responsables de la compensation en fonction de la concentration de bore implanté.

[Annealing temperature to have a complete disparition of the compensation versus the implanted boron concentration.] 


\section{Implantation de fer dans l'InP de type $\mathbf{N}$.}

Des implantations à $800 \mathrm{keV}$ de fer à différentes doses ont été faites dans des substrats de type $n$ $\left(n=4 \times 10^{16} \mathrm{e}^{-} \cdot \mathrm{cm}^{-3}\right)$. Après recuit nous avons déposé sur ces échantillons des contacts ohmiques sur la face arrière et des plots d'or sur la face implantée. Pour de telles structures réalisées, la capacité à OV varie fortement avec la dose implantée et la température de recuit. D'autre part, à partir de ces structures, quand la résistance de la couche implantée est forte, on peut évaluer cette résistance, et ainsi accéder à son épaisseur et à sa résistivité. Sur la figure 6 , nous présentons la variation de l'épaisseur de la couche de haute résistivité déduite des mesures capacitives à $\mathrm{OV}$, en fonction des doses d'implantation et pour diverses températures de recuit.

Sur ces couches nous notons que les épaisseurs sont croissantes avec la dose d'implantation et avec la température de recuit jusqu'à $700^{\circ} \mathrm{C}$. Pour les fortes températures de recuit, la résistivité moyenne de la couche implantée est de l'ordre de $10^{7} \Omega \mathrm{cm}$. Cette forte résistivité est bien induite par l'implantation de fer, car à ces températures de recuit la compensation induite par les défauts primaires a totalement disparue quand l'impureté implantée est inactive dans l'InP (cas du bore, oxygène néon, argon). D'autre part, la grande différence d'épaisseur pour les recuits à $600^{\circ} \mathrm{C}$ et $700^{\circ} \mathrm{C}$ montre que l'activation des centres de compensation induisant la haute résistivité se produit à haute température et ces centres de compensation formés par l'implantation de fer restent stables jusqu'à $750^{\circ} \mathrm{C}$ (malgré une légère diminution, non expliquée, de l'épaisseur de la couche semi-isolante, à cette température). Par microanalyse ionique (SIMS), les profils de fer, dans ces mêmes échantillons implantés fer et recuits, ont été tracés (Fig. 7). Lors des recuits, le fer

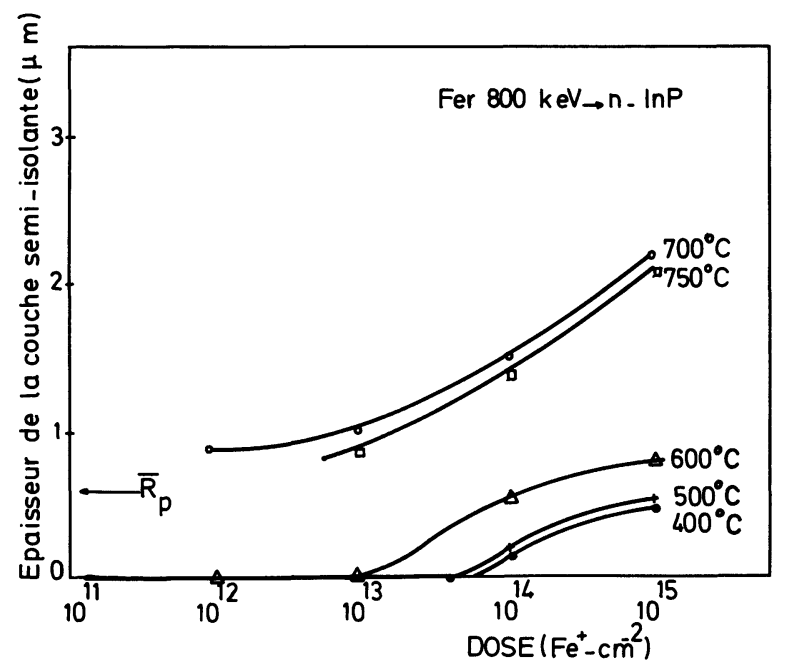

Fig. 6. - Epaisseur de la couche de haute résistivité après implantation de fer et recuit à haute température. $\bar{R}_{\mathrm{p}}$ est le parcours projeté des ions implantés.

[Thickness of the high resistivity layer after iron implantation and a high temperature annealing.]

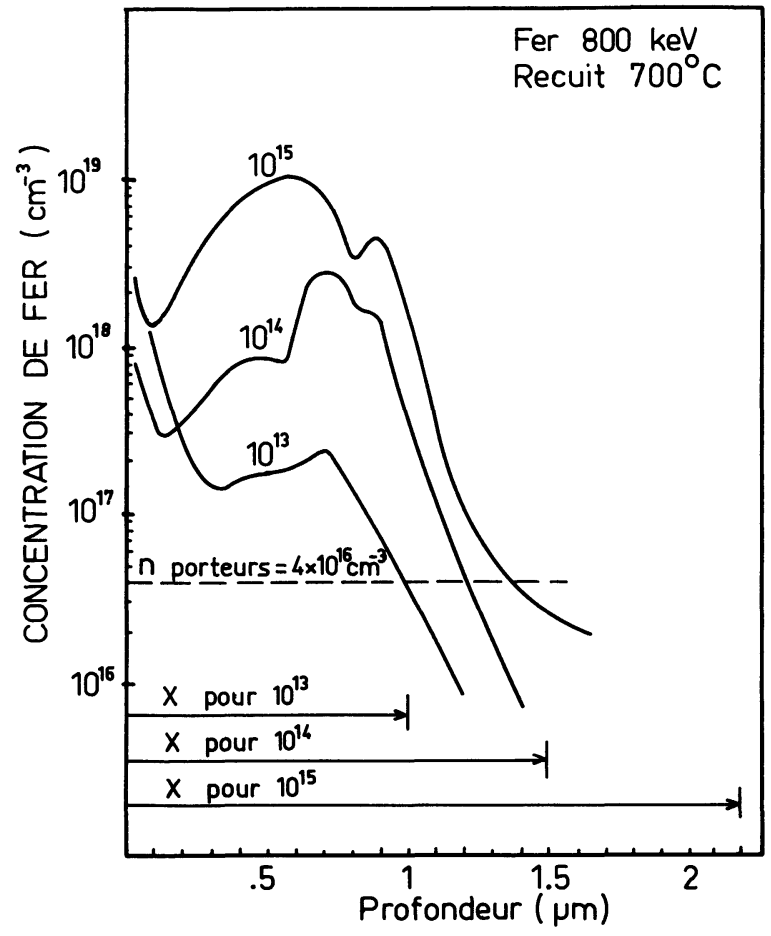

Fig. 7. - Profils de fer après implantation à $800 \mathrm{keV}$ et recuit à $700{ }^{\circ} \mathrm{C}$. Pour chaque dose, l'épaisseur $x$ de la couche de haute résistivité est indiquée. Le substrat implanté était de type $\mathrm{n}$ avec $n=4 \times 10^{16} \mathrm{e}^{-} \cdot \mathrm{cm}^{-3}$.

[After annealing at $700^{\circ} \mathrm{C}$, iron depth profiles. For each dose, the thickness $x$ of the high resistivity is shown. The implanted substrate was n-type $\left(n=4 \times 10^{16} \mathrm{e}^{-} . \mathrm{cm}^{-3}\right)$.]

se redistribue et la forme du profil est complexe [15] mais on ne note pas de queue de diffusion notable. La croissance importante, avec la dose, de l'épaisseur de la couche de haute résistivité n'est pas due à une diffusion de fer (comme l'avait précédemment suggéré Donnelly et Hurwitz [12] et on note d'ailleurs qu'il n'y a pas de fer, au moins à des concentrations supérieures à $4 \times 10^{16} \mathrm{~cm}^{-3}$ au-delà de $1,3 \mu \mathrm{m}$ pour la dose de $10^{15} \mathrm{Fe}^{+} \mathrm{cm}^{-2}$. Dans les régions à haute résistivité où la concentration de fer reste faible, la compensation n'est pas due au fer, mais serait plutôt due à une modification dans la distribution de défauts (diffusion de défauts?) ou d'impuretés (migration d'impuretés autres que le fer ?), et cette modification serait quand même induite par l'implantation de l'impureté fer.

\section{Conclusion.}

L'augmentation de résistivité de l'InP après bombardement ionique est induite par la formation de centres de compensation. Ces centres de compensation sont formés à partir des atomes de la matrice déplacés, In et $\mathbf{P}$, lors dês chocs nucléaires. Quelle que soit la nature de l'ion implanté, on obtient toujours cette augmentation de résistivité l'efficacité de compensation de l'ion incident dépend de sa masse : plus l'ion incident est lourd, plus le nombre d'atomes déplacé est grand et plus l'efficacité de compensation est grande. 
La résistivité maximale obtenue dans l'InP de type n est de $5 \times 10^{3} \Omega \mathrm{cm}$, résultat comparable à celui publié par Donnelly et al. [10]. Cette résistivité maximale, pour les fortes doses d'implantation, dépend du couple dose-température de recuit (voir Fig. 4), ce qui laisse penser que la résistivité maximale correspond à une certaine densité de défauts. Quand la densité de défauts est trop importante, il faut en guérir un certain nombre pour accéder à la compensation maximale.

La compensation induite par le déplacement des atomes de la matrice disparaît aux fortes températures de recuit. La température nécessaire pour "guérir " les échantillons implantés dépend de la dose d'implantation. Au-delà de cette température, et pour les ions neutres vis-à-vis de l'InP, la couche implantée retrouve la résistivité qu'elle avait avant le bombardement. Au vu de ces résultats, une température de $650^{\circ} \mathrm{C}$ serait la température la plus basse nécessaire pour étudier les dopages obtenus par implantation ionique. Les impuretés bore et oxygène dans l'InP se comportent comme les impuretés neutres hydrogène, néon et argon, elles n'ont aucune activité électrique dans l'InP et ce contrairement à ce qui se passe dans le GaAs.

Le fer implanté induit une forte compensation dans l'InP après recuit à haute température. Les résistivités obtenues sont plus grandes que $10^{7} \Omega \mathrm{cm}$. Une comparaison entre les profils chimiques de fer et les propriétés électriques nous laissent supposer que la compensation n'est pas due uniquement à une compensation par le fer, mais aussi à des défauts qui seraient induits par la présence du fer.

\section{Bibliographie}

[1] Foyt, A. G., Lindley, W. T., Wolfe, C. M. et DonNELLy, P. N., Solid State Electronics 12 (1969) 209.

[2] Dyment, J. C., North, J. C. et D'Asaro, L. R., J. Appl. Phys. 44 (1973) 207.

[3] Favennec, P. N. et Diguet, D., Appl. Phys. Lett. 23 (1973) 546.

[4] Rao, E. V. K., Duhamel, N., Favennec, P. N., L'HaRIDON, H., Ion Implantation in Semiconductors (Plenum, New York) 1965 p. 77.

[5] Berth, M., Cathelin, M. et Durand, G., Tech. Dig. IEDM (1977) 201.

[6] Toulouse, B., Favennec, P. N., Guivarc'H, A. et Pelous, G., Inst. Phys. Conf. 45 (1979) 501.

[7] Favennec, P. N., Pelous, G., Binet, M. et Baudet, P., Ion Implantation in Semiconductors (Plenum, New York) 1974 p. 621.
[8] Favennec, P. N., J. Appl. Phys. 47 (1976) 2532.

[9] Deveaud, B. et Favennec, P. N., Solid State Commun. 24 (1976) 473.

[10] Donnelly, J. P. et Hurwitz, C. E., Solid State Electron. 20 (1977) 727.

[11] Davies, D. E., Lorenzo, J. P. et Deane, M. L., Appl. Phys. Lett. 31 (1977) 256.

[12] Donnelly, J. P. et Hurwitz, C. E., Solid State Electron. 21 (1978) 475.

[13] Flocht, M. W. et Schwartz, B., Appl. Phys. Lett. 42 (1983) 970.

[14] Toulouse, B., Favennec, P. N., GuivarC'H, A., Pelous, G., Inst. Phys. Conf. Ser. 45 (1979) 501.

[15] Gauneau, M., L'Haridon, H., Rupert, A. et Salvi, M., J. Appl. Phys. 52 (1982) 6823. 